\title{
Electron capture from helium atoms by fast protons
}

\author{
I Mančev ${ }^{1}$, V Mergel $^{2}$ and L Schmidt ${ }^{2}$ \\ ${ }^{1}$ Department of Physics, Faculty of Sciences and Mathematics, University of Niš, PO Box 224, \\ 18000 Niš, Serbia \\ ${ }^{2}$ Institut für Kernphysik, Universität Frankfurt, Auger-Euler-Straße 6, D-60486 Frankfurt am \\ Main, Germany
}

Received 22 January 2003

Published 13 June 2003

Online at stacks.iop.org/JPhysB/36/2733

\begin{abstract}
The differential and total cross sections for single charge exchange in $\mathrm{p}+\mathrm{He}$ collisions have been calculated by means of the Born distorted wave (BDW$4 \mathrm{~B})$ approximation. The BDW-4B model is a four-body quantum-mechanical distorted-wave theory. The computations of total cross sections are carried out at impact energies ranging from $40 \mathrm{keV}$ to $15 \mathrm{MeV}$. The differential cross sections are calculated at impact energies covering 50, 100, 150, 200, 293, 300 and $400 \mathrm{keV}$. The theoretical results obtained for the differential and total cross sections are compared with the available experimental data and good agreement is found.
\end{abstract}

\section{Introduction}

Single electron capture in collisions between protons and helium atoms at intermediate and high impact energies has been the subject of many theoretical and experimental articles. Thus far, the differential cross sections (DCS) for $p+$ He collisions have been theoretically evaluated by means of various methods. For example, the three-body continuum distorted wave (CDW3B) model was applied by Belkić and Salin [1] in p + He collisions at $293 \mathrm{keV}$. They clearly showed the discrepancy between DCS obtained with and without the Coulomb internuclear repulsion. The same method was utilized by Rivarola et al [2] for calculating the DCS at higher impact energies $(2.82,5.42$ and $7.40 \mathrm{MeV})$. The second Born approximation employing a free-particle Green function has been used in [3, 4]. At high energies the DCS for capture in $\mathrm{p}+\mathrm{He}$ collisions have also been calculated by means of a second-order Faddeev scattering formalism [5], as well as using the strong potential Born method [6]. The calculations of the DCS at intermediate energies employing the close-coupling method have been carried out by Slim et al [7] and Martin et al [8]. The experimental data for the DCS in p + He collisions have been measured by Martin et al [8] (at incident proton energies of 25, 30, 50 and $100 \mathrm{keV}$ ) by Bratton et al [9] (at the energy $293 \mathrm{keV}$ ), by Horsdal-Pedersen et al [10] (at the impact energies 2.82, 5.42 and $7.40 \mathrm{MeV}$ ). In a very recent work Mergel et al [11] using the cold-target recoil ion momentum spectroscopy (COLTRIMS) technique have systematically measured the DCS for some ten impact energies between 150 and $1400 \mathrm{keV}$. All of the above 
quoted theoretical considerations are within the framework of the independent-electron model, in which the state of the non-captured electron is kept frozen and, as such, eliminated from the $T$-matrix elements. Single electron capture from a helium-like atom (ion) by a bare nucleus is a purely four-body problem. Consequently, evaluations of the DCS in the four-body framework of scattering theory are preferable, especially since this has not been previously assessed. Therefore, in the present work the differential and total cross sections will be presented for single charge exchange in $\mathrm{p}+$ He collisions obtained by means of the four-body Born distorted wave (BDW) approximation [12]. For single electron capture, the BDW model has been introduced by Mančev in order to evaluate intermediate ionization continua [12]. This theory has been successfully applied [12] for describing single charge exchange in $\alpha+$ He collisions. The computations of the total cross sections $Q_{i f}^{\mathrm{BDW}}$ performed in [12] showed a very good agreement with experimental data at intermediate and high impact energies.

Recently, Belkić et al [13] devised a strict extension of Cheshire's [14] three-body CDW theory to the four-body problem and investigated single charge exchange without further approximation. Such a CDW-4B model has subsequently been applied to calculation of the total cross sections and evaluation of the correlation effects in different processes $[15,16]$. This CDW-4B model and other leading distorted wave theories and computational methods for high-energy rearranging collisions of nuclei with atomic targets have recently been critically reviewed by Belkić [17].

The prior form of the transition amplitude in the CDW-4B method is given by the matrix elements $T_{i f}^{\mathrm{CDW}}=\left\langle\chi_{f}^{-}\left|U_{i}\right| \chi_{i}^{+}\right\rangle$. Here, the distorting potential is denoted by $U_{i}$ and the distorted waves $\chi_{i, f}^{ \pm}$are expressed as a product of the corresponding bound state wavefunctions with the two Coulomb waves. The pure electronic continuum intermediate states are included in both channels. On the other hand, in the boundary-corrected four-body first Born (CB1-4B) model for single charge exchange $[18,19]$ the scattering state vectors $\chi_{i, f}^{\mathrm{CB} 1 \pm}=\Phi_{i, f}^{ \pm}$are given by the product of the unperturbed channel states and the logarithmic distortion phase factors, due to the Coulomb long-range remainders of the perturbation potentials. As such, in the CB1 approximation, electronic continuum intermediate states are completely ignored.

In the BDW-4B model the initial distorted wavefunction $\chi_{i}^{+}$and the associated perturbation potential $U_{i}$ contained in the transition amplitude coincide with the corresponding quantities of the CDW-4B model. The treatment of the exit channel, however, is identical to that of the CB1$4 \mathrm{~B}$ model. Hence, the BDW-4B approximation is of the hybrid type which is intermediate to the CDW-4B and CB1-4B approaches. According to the BDW-4B theory, the captured electron is treated in an asymmetrical manner in the entrance and exit channels.

The BDW-4B model preserves the four-body nature of the problem and satisfies the correct boundary conditions in the entrance and exit channels. Double charge exchange has already been investigated by means of similar four-body distorted-wave approaches [20-22].

In the present work the numerical computations of the DCS for single electron capture in p + He collisions are performed at 50,100, 150, 200, 293, 300 and $400 \mathrm{keV}$. The computations of the total cross sections are carried out for the same reaction for a very wide range of impact energies, from $40 \mathrm{keV}$ to $15 \mathrm{MeV}$. The validity of the reported theoretical results is assessed in comparison with the available experimental data.

Atomic units will be used throughout unless otherwise stated.

\section{Theory}

We shall consider single electron capture in collisions between completely stripped projectiles and helium-like targets, i.e.

$$
Z_{P}+\left(Z_{T} ; \mathrm{e}_{1}, \mathrm{e}_{2}\right) \longrightarrow\left(Z_{P} ; \mathrm{e}_{1}\right)+\left(Z_{T} ; \mathrm{e}_{2}\right)
$$


where $Z_{P}$ and $Z_{T}$ are the nuclear charges of the projectile and the target. Let $\vec{x}_{k}\left(\vec{s}_{k}\right)$ be the relative vector of the $k$ th electron with respect to the nucleus of the target (projectile) $(k=1,2)$. The internuclear vector directed from the target nucleus to the projectile nucleus will be denoted by $\vec{R}$, whereas the distance between the electrons is given by $r_{12}$. In the entrance channel, it is convenient to introduce $\vec{r}_{i}$ as a relative vector of $Z_{P}$ with respect to the centre of mass of $\left(Z_{T} ; \mathrm{e}_{1}, \mathrm{e}_{2}\right)$. Symmetrically, $\vec{r}_{f}$ will denote the relative vector between the centre of masses of the $\left(Z_{T} ; \mathrm{e}_{2}\right)$ and $\left(Z_{P} ; \mathrm{e}_{1}\right)$ in the exit channel of process $(2.1)$. The reduced mass of the two aggregates $\left(Z_{P} ; \mathrm{e}_{1}\right)$ and $\left(Z_{T} ; \mathrm{e}_{2}\right)$ will be denoted by $\mu_{f}$, whereas $\mu_{i}$ will label the reduced mass of the projectile and the target. Further, $m_{P}\left(m_{T}\right)$ will denote the mass of the $P(T)$ nucleus.

The treatment of the entrance channel is identical to that of the CDW-4B method, with the same distorted wave and the associated distorting potential. Namely, the initial distorted wave is expressed as a product of the initial bound state $\varphi_{i}\left(\vec{x}_{1}, \vec{x}_{2}\right)$ with the two Coulomb waves (one for the electronic and the other for the nuclear motion)

$\chi_{i}^{+}=N^{+}\left(v_{P}\right) \mathcal{N}^{+}(v) \mathrm{e}^{\mathrm{i} \vec{k}_{i} \cdot \vec{r}_{i}} \varphi_{i}\left(\vec{x}_{1}, \vec{x}_{2}\right)_{1} F_{1}\left(\mathrm{i} v_{P}, 1, \mathrm{i} v s_{1}+\mathrm{i} \vec{v} \cdot \vec{s}_{1}\right)_{1} F_{1}\left(-\mathrm{i} v, 1, \mathrm{i} k_{i} r_{i}-\mathrm{i} \vec{k}_{i} \cdot \vec{r}_{i}\right)$.

Here, the symbol ${ }_{1} F_{1}(a, b, c)$ denotes the conventional confluent hypergeometric function and $N^{+}\left(v_{P}\right)=\Gamma\left(1-\mathrm{i} v_{P}\right) \mathrm{e}^{\pi v_{P} / 2}, \mathcal{N}^{+}(v)=\Gamma(1+\mathrm{i} v) \mathrm{e}^{-\pi v_{P} / 2}$, with $v_{P}=Z_{P} / v$ and $v=Z_{P}\left(Z_{T}-1\right) / v$. The quantity $\vec{k}_{i}$ represents the initial wavevector, whereas $v$ is the incident velocity. The distorted wave $\chi_{i}^{+}$obeys the correct boundary condition. The corresponding perturbation potential $U_{i}$, which explicitly appears in the prior transition amplitude, is given by

$$
U_{i}=V_{i}-W_{i} \equiv Z_{P}\left(\frac{1}{R}-\frac{1}{s_{2}}\right)-\vec{\nabla}_{x_{1}} \ln \varphi_{i}\left(\vec{x}_{1}, \vec{x}_{2}\right) \cdot \vec{\nabla}_{s_{1}},
$$

where $V_{i}$ is the perturbation potential in the entrance channel: $V_{i}=Z_{P} Z_{T} / R-Z_{P} / s_{1}-Z_{P} / s_{2}$, while $W_{i}$ is the distorting potential operator.

The distorted wave $\chi_{f}^{-}$in the exit channel is obtained by the use of the distorting potential in the form $W_{f}=\left(Z_{P}-1\right)\left(Z_{T}-1\right) / r_{f}$, so that

$$
\chi_{f}^{-}=\mathcal{N}^{+}\left(v_{f}\right) \varphi_{P}\left(\vec{s}_{1}\right) \varphi_{T}\left(\vec{x}_{2}\right) \mathrm{e}^{-\mathrm{i} \vec{k}_{f} \cdot \vec{r}_{f}} F_{1}\left(\mathrm{i} v_{f}, 1,-\mathrm{i} k_{f} r_{i}-\mathrm{i} \vec{k}_{f} \cdot \vec{r}_{i}\right),
$$

where $v_{f}=\left(Z_{P}-1\right)\left(Z_{T}-1\right) / v$ and the final wavevector is labelled by $\vec{k}_{f}$. The bound state wavefunctions of the atomic systems $\left(Z_{P}, \mathrm{e}_{1}\right)$ and $\left(Z_{T}, \mathrm{e}_{2}\right)$ are denoted by $\varphi_{P}\left(\vec{s}_{1}\right)$ and $\varphi_{T}\left(\vec{x}_{2}\right)$, respectively. The distorted wave $\chi_{f}^{-}$possesses the proper asymptotic behaviour.

The prior form of the transition amplitude as a function of the transversal component of the momentum transfer $(\vec{\eta})$, in the BDW model is

$$
T_{i f}=\left\langle\chi_{f}^{-}\left|U_{i}\right| \chi_{i}^{+}\right\rangle \text {. }
$$

The explicit expression for this amplitude is

$$
\begin{aligned}
T_{i f}(\vec{\eta})=N^{+}\left(v_{P}\right) \iiint \mathrm{d} \vec{x}_{1} \mathrm{~d} \vec{x}_{2} \mathrm{~d} \vec{R} \mathrm{e}^{\mathrm{i} \vec{\alpha} \cdot \vec{s}_{1}+\mathrm{i} \vec{\beta} \cdot \vec{x}_{1}} \mathcal{L}(R) \varphi_{P}^{*}\left(\vec{s}_{1}\right) \varphi_{T}^{*}\left(\vec{x}_{2}\right) \\
\times\left[V\left(R, s_{2}\right) \varphi_{i}\left(\vec{x}_{1}, \vec{x}_{2}\right)_{1} F_{1}\left(\mathrm{i} v_{P}, 1, \mathrm{i} v s_{1}+\mathrm{i} \vec{v} \cdot \vec{s}_{1}\right)\right. \\
\left.-\vec{\nabla}_{x_{1}} \varphi_{i}\left(\vec{x}_{1}, \vec{x}_{2}\right) \cdot \vec{\nabla}_{s_{1} 1} F_{1}\left(\mathrm{i} v_{P}, 1, \mathrm{i} v s_{1}+\mathrm{i} \vec{v} \cdot \vec{s}_{1}\right)\right] \\
\equiv T_{R}-T_{\nabla},
\end{aligned}
$$

where

$$
\begin{aligned}
& V\left(R, s_{2}\right)=Z_{P}\left(1 / R-1 / s_{2}\right), \quad \vec{\alpha}=\vec{\eta}-v^{-\hat{v}} \\
& \vec{\beta}=-\vec{\eta}-v^{+} \hat{\vec{v}}, \quad v^{ \pm}=v / 2 \pm\left(\varepsilon_{i}-\varepsilon_{f}\right) / v
\end{aligned}
$$


The quantities $\varepsilon_{i}$ and $\varepsilon_{f}$ represent the initial and final bound-state energies. The function $\mathcal{L}(R)$ is defined by

$$
\begin{gathered}
\mathcal{L}(R)=\left[\left(\mathcal{N}^{+}\right)\left(v_{f}\right)\right]^{*} \mathcal{N}^{+}(v)_{1} F_{1}\left(-\mathrm{i} v_{f}, 1, \mathrm{i} k_{f} r_{i}+\mathrm{i} \vec{k}_{f} \cdot \vec{r}_{i}\right)_{1} F_{1}\left(-\mathrm{i} v, 1, \mathrm{i} k_{i} r_{i}-\mathrm{i} \vec{k}_{i} \cdot \vec{r}_{i}\right) \\
\simeq\left(k_{f} r_{i}+\vec{k}_{f} \cdot \vec{r}_{i}\right)^{\mathrm{i} v_{f}}\left(k_{i} r_{i}-\vec{k}_{i} \cdot \vec{r}_{i}\right)^{\mathrm{i} v}, \quad\left(r_{i} \rightarrow \infty\right) .
\end{gathered}
$$

Such a replacement of the Coulomb scattering waves for relative motion of the heavy particles by their asymptotic forms has been used and discussed in a number of articles (see, for example, [17, 23, 39-41]). A similar replacement constitutes the main difference between the Coulomb-Born approximation and boundary-corrected first Born method (CB1). The essence of the Coulomb-Born method [39] lies in the fact that the long-range Coulomb interaction between the colliding partners in the entrance and exit channels is taken into account by using the full Coulomb waves for the relative motion of the heavy particles. The CB1 method can be obtained with the replacement of the outgoing and incoming scattering wavefunctions by their asymptotic forms. Numerous investigations [36, 42-45] have confirmed that the CB1 approximation is an accurate theory for rearrangement collisions in reproducing the experimental data at intermediate and high impact energies. We now resort to the well-known eikonal hypothesis, which assumes that $k_{i}$ acquires large values. For heavy-particle collisions, we have $\mu_{i} \gg 1$ and, hence, $k_{i}$ is large even at very small incident velocities $v_{i}$ (say, of the order of $0.01 \mathrm{au}$ ). Due to their large mass, heavy projectiles are only slightly deflected from the initial direction and the scattering predominantly takes place in a narrow forward cone, which implies that $\hat{\vec{k}}_{i} \simeq \hat{\vec{k}}_{f}$. Therefore, in the mass limit $m_{P, T} \gg 1$, for the initial and final heavy-particle velocities $\vec{v}_{i}=\vec{k}_{i} / \mu_{i}$ and $\vec{v}_{f}=\vec{k}_{f} / \mu_{f}$ we can write $\vec{v}_{i} \simeq \vec{v}_{f} \equiv \vec{v}$. This mass limit also justifies the replacement of $\vec{r}_{i}$ by $\vec{R}$. Thus, such an eikonal hypothesis permits a consistent reduction of the function $\mathcal{L}(R)$ to the following simplified form:

$$
\begin{aligned}
\mathcal{L}(R) & \simeq(v R-\vec{v} \cdot \vec{R})^{\mathrm{i} Z_{P}\left(Z_{T}-1\right) / v}(v R+\vec{v} \cdot \vec{R})^{\mathrm{i}\left(Z_{P}-1\right)\left(Z_{T}-1\right) / v} \\
& =\rho^{2 \mathrm{i} Z_{P}\left(Z_{T}-1\right) / v}(v R+\vec{v} \cdot \vec{R})^{-\mathrm{i} \xi}, \quad \xi=\left(Z_{T}-1\right) / v,
\end{aligned}
$$

where the unimportant phase factors of the unit moduli are dropped. Here, $\vec{\rho}$ is the component of the vector of the internuclear distance perpendicular to the $Z$ axis $(\vec{R}=\vec{\rho}+\vec{Z})$. The factor $\rho^{2 \mathrm{i} Z_{P}\left(Z_{T}-1\right) / v}$ has no influence on the total cross sections and may be omitted. However, it ought to be recalled that this factor does not disappear from the DCS. The method of analytical calculation from [12] provides the matrix elements $T_{i f}(\vec{\eta})$ in terms of two-dimensional real quadratures.

The DCS is defined by the relation (see, for example, [23])

$$
\frac{\mathrm{d} Q_{i f}}{\mathrm{~d} \Omega}=\frac{\mu_{i} \mu_{f}}{4 \pi^{2}} \frac{k_{f}}{k_{i}}\left|T_{i f}(\vec{\eta})\right|^{2} \quad\left(\frac{a_{0}^{2}}{s r}\right) .
$$

The ratio $k_{f} / k_{i}$ of the magnitudes of the final and initial wavevectors is close to unity, since the heavy projectiles scatter mainly in the forward direction at high impact energies. The scattering angle $\theta$ is identified from the relation $\eta=2 \mu_{P T} v \sin (\theta / 2)$, where $\mu_{P T}$ is the reduced mass of the projectile and target nucleus. Calculation of the angular distributions $\mathrm{d} Q_{i f} / \mathrm{d} \Omega$ becomes particularly convenient in the BDW approximation when a proton is used as a projectile, i.e. $Z_{P}=1$. This is because the products of the logarithmic Coulomb phase factors from the function $\mathcal{L}(R)$ will be reduced to the single term $(v R-\vec{v} \cdot \vec{R})^{\mathrm{i} \xi}$. Hence, a trivial modification of the sign of the parameter $\xi$, as well as of the sign of the term $\vec{v} \cdot \vec{R}$, will enable us to obtain both differential (with factor $\left.(v R-\vec{v} \cdot \vec{R})^{\mathrm{i} \xi}\right)$ and total cross sections (with factor $(v R+\vec{v} \cdot \vec{R})^{-\mathrm{i} \xi}$ ) from the same algorithm associated with the BDW-4B method. It should be noted that the computation of the DCS in the CDW-4B method becomes a much more 
laborious task, involving highly oscillating Bessel functions and evaluation of the transition amplitude $\mathcal{A}_{i f}(\vec{\rho})$ by means of the Fourier transform $\mathcal{A}_{i f}(\vec{\rho})=(2 \pi)^{-1} \int \mathrm{d} \vec{\kappa} \mathrm{e}^{\mathrm{i} \vec{\kappa} \cdot \vec{\rho}} T_{i f}^{\mathrm{CDW}}(\vec{\kappa})$.

The DCS in the centre of mass $(\mathrm{cm})$ system is given by the well-known formula [23]

$$
\frac{\mathrm{d} Q_{i f}^{\mathrm{CDW}}}{\mathrm{d} \Omega}=\left|\mathrm{i} v \mu_{P T} \int_{0}^{\infty} \mathrm{d} \rho \rho^{1+2 \mathrm{i} v_{P T}} \mathcal{A}_{i f}(\rho) J_{m_{i}-m_{f}}(\rho \eta)\right|^{2} \quad\left(\frac{a_{0}^{2}}{s r}\right),
$$

where $v_{P T}=Z_{P} Z_{T} / v$ and $J_{v}(z)$ is the Bessel function of the first order and $v$ kind, $m_{i}$ and $m_{f}$ are the projection of the angular momentum on the $Z$ axis, which is chosen in the incidence direction of the projectile. In addition to the inevitable three-dimensional integrations in the expression of Belkić et al [13] for $T_{i f}^{\mathrm{CDW}}(\vec{\eta})$, one would have to perform another complicated quadrature over $\kappa$ of the type

$$
\mathcal{A}_{i f}(\rho)=\int_{0}^{\infty} \mathrm{d} \kappa \kappa J_{m_{i}-m_{f}}(\kappa \rho) T_{i f}^{\mathrm{CDW}-4 \mathrm{~B}}(\kappa) .
$$

This means that the DCS in the CDW-4B approximation are obtained through the fivedimensional integrations over integrands, weighted with the two highly oscillating Bessel functions. The same drawback is also encountered in the continuum distorted wave eikonal initial state (CDW-EIS) approximation (see, for example, [24, 25]). Due to this difficulty alone, encountered in the CDW-4B and CDW-EIS models, it is advisable to search for alternative distorted wave methods along the lines of the BDW-4B and other similar fourbody approximations.

The physical interpretation of the $T$-matrix element can be done in the following manner. The incident particle scatters on each of the three constituents of the target $\left(Z_{T} ; \mathrm{e}_{1}, \mathrm{e}_{2}\right)$. The BDW-4B model allows the projectile to separately distort the nuclear and electronic motions through the additive Coulombic interactions. Thus, the interaction of the $Z_{P}$ with the electron $\mathrm{e}_{1}$ leads to ionization of the target. The ionized electron propagates in the Coulomb field of $Z_{P}$ in a particular eikonal direction with the momentum $\vec{k}_{1}=\vec{v}$. The capture of the $\mathrm{e}_{1}$ electron occurs from this intermediate ionizing state (capture from continuum), because the electron is travelling together with the projectile in the same direction, and the attractive force between $Z_{P}$ and $e_{1}$ is sufficient to bind them together into a new hydrogen-like atomic system $\left(Z_{P} ; \mathrm{e}_{1}\right)$. The residual target $\left(Z_{T} ; \mathrm{e}_{2}\right)$ takes the extra energy and recoils, thus preserving the energy conservation law and thus enabling capture to take place. This is a quantum version of the well-known Thomas classical double scattering. Further, collision between the projectile $Z_{P}$ and the residual target $\left(Z_{T} ; \mathrm{e}_{2}\right)$ results in accumulation of the Coulomb phase factor $\exp \left\{\mathrm{i}\left[Z_{P}\left(Z_{T}-1\right) / v\right] \ln (v R-\vec{v} \cdot \vec{R})\right\}$. On the other hand, in the exit channel, the residual $\operatorname{target}\left(Z_{T} ; \mathrm{e}_{2}\right)$ interacts with the newly formed atom or ion $\left(Z_{P} ; \mathrm{e}_{1}\right)$ (both considered as point charges $)$, thus accumulating the phase factor $\exp \left\{\mathrm{i}\left[\left(Z_{P}-1\right)\left(Z_{T}-1\right) / v\right] \ln (v R+\vec{v} \cdot \vec{R})\right\}$ due to the asymptotic residual Coulomb interaction $W_{f}^{D}=\left(Z_{P}-1\right)\left(Z_{T}-1\right) / R$. Here, no explicit electronic distortion factors are taken into account.

Hence, according to the BDW model, the electron $\mathrm{e}_{1}$ intermediately finds itself in the onshell continuum state of the projectile nucleus before actual capture takes place. On the other hand, in the corrected first Born (CB1-4B) model, the electron $\mathrm{e}_{1}$ is free in the intermediate stage of collision involving single charge exchange.

\section{Results and discussion}

We apply the BDW-4B approximation to compute both the differential and total cross sections at intermediate and high impact energies for single electron capture by protons from helium:

$$
\mathrm{p}+\mathrm{He} \longrightarrow \mathrm{H}+\mathrm{He}^{+} \text {. }
$$


The present computations are performed by using the uncorrelated one-parameter wavefunction for the initial bound state of the target: $\varphi_{i}\left(\vec{x}_{1}, \vec{x}_{2}\right)=\left(\gamma^{3} / \pi\right) \mathrm{e}^{-\gamma\left(x_{1}+x_{2}\right)}$, where $\gamma$ is the effective charge $\gamma=Z_{T}-5 / 16$. The proposed BDW theory describes only capture to the $1 \mathrm{~s}$ final state. We suppose that the inclusion of the capture in excited states does not significantly influence the cross sections and contributions from these states are roughly approximated by an overall multiplicative factor 1.202 according to the Oppenheimer scaling law. Such an assumption is based on the following facts. There are a number of articles in which formation of the atomic hydrogen in excited states in $\mathrm{p}+\mathrm{He}$ collisions at intermediate and high impact energies has been investigated [36, 42, 46-50]. Such cross sections for capture into individual excited states have been determined by means of various models, for instance: CB1-3B [36, 42], CDW-3B [46], symmetric eikonal approximation [47], eikonal impulse approach [48], target continuum distorted wave (TCDW) [49], continuum distorted wave eikonal initial (final) state CDW-EIS (CDW-EFS) [50], a coupled-state approach using a Sturmian bases [38], etc. These calculations, based upon entirely different theoretical frameworks, provide individual excited-state cross sections and show that, for $\mathrm{p}+\mathrm{He}$ collisions, an explicit inclusion of excited states does not differ appreciably from the corresponding ground-to-ground state capture cross section pre-multiplied by 1.202. These theoretical data are also tested against the numerous measurements. Of course, if $Z_{P}$ increases, the capture into excited states becomes more important.

Next, we turn our attention to the terms in the perturbation potential $U_{i}$, which appears in the transition amplitude. There are two distinct contributions to the matrix elements $T_{i f}$ from the perturbation in the entrance channel $U_{i}=V\left(R, s_{2}\right)-\vec{\nabla}_{x_{1}} \ln \varphi_{i}\left(\vec{x}_{1}, \vec{x}_{2}\right) \cdot \vec{\nabla}_{s_{1}}$, given in equation (2.3). In the term $V\left(R, s_{2}\right)=Z_{P}\left(1 / R-1 / s_{2}\right)$, the interaction $-Z_{P} / s_{2}$ is the Coulomb potential between the projectile $Z_{P}$ and the passive electron $\mathrm{e}_{2}$. The asymptotic tail of this potential is $-Z_{P} / R$, since $s_{2} \rightarrow R$ as $R \rightarrow \infty$, so that the potential $V\left(R, s_{2}\right)$ behaves as a short range interaction at large values of the inter-aggregate separation. This can easily be checked by using the Taylor series expansion for $1 / s_{2}=1 /\left|\vec{R}-\vec{x}_{2}\right|$ around $R$ : $V\left(R, s_{2}\right)=Z_{P}\left[1 / R-1 /\left|\vec{R}-\vec{x}_{2}\right|\right]=Z_{P}\left[1 / R-\left(1 / R-\vec{R} \cdot \vec{x}_{2} / R^{3}+\cdots\right)\right] \simeq Z_{P} \vec{R} \cdot \vec{x}_{2} / R^{3}$. The small value of the $x_{2}$ coordinate justifies such a development, since the electron $\mathrm{e}_{2}$ remains bound to the target nucleus. The potential $-Z_{P} / s_{2}$ in the $T$ matrix plays the role of a perturbation which causes capture of the electron $\mathrm{e}_{1}$. Therefore, the active electron $\mathrm{e}_{1}$ can be captured by the projectile without its direct interaction with $Z_{P}$. Instead, the passive electron $\mathrm{e}_{2}$ interacts directly with $Z_{P}$ and the subsequent transfer of $\mathrm{e}_{1}$ to the projectile is made possible through the static $\mathrm{e}_{1}-\mathrm{e}_{2}$ correlation in the ground state of the target $\left(Z_{T} ; \mathrm{e}_{1}, \mathrm{e}_{2}\right)$. This latter effect is termed the 'static correlation', since it exists in any two-electron system as one of its spectroscopic features without any reference to collisions. Hence, a part of the energy received by the electron $\mathrm{e}_{2}$ in its collision with $Z_{P}$ could be sufficient to accomplish the transfer of $\mathrm{e}_{1}$ to the projectile, provided that the $\mathrm{e}_{1}-\mathrm{e}_{2}$ correlation is active. This is possible as explained by the following argument. Using the relation $\vec{R}=\vec{x}_{1}-\vec{s}_{1}=\vec{x}_{2}-\vec{s}_{2}$ we can express the $s_{2}$ coordinate through the inter-electronic coordinate $\vec{r}_{12}=\vec{x}_{1}-\vec{x}_{2}=\vec{s}_{1}-\vec{s}_{2}$ by means of relation $\vec{s}_{2}=\vec{s}_{1}-\vec{r}_{12}$. The electron $\mathrm{e}_{1}$ is captured in the final bound state $\varphi_{P}\left(\vec{s}_{1}\right)$ in the reaction (2.1) and, therefore, $s_{1}$ is of the order of the Bohr radius $a_{0}$. For such small values of $s_{1}$, we can develop $1 / s_{2}$ in the Taylor expansion: $1 / s_{2}=1 /\left|\vec{r}_{12}-\vec{s}_{1}\right|=1 / r_{12}-\vec{r}_{12} \cdot \vec{s}_{1} / r_{12}^{3}+\cdots$. From here we can see that the potential $-Z_{P} / s_{2}$ contains information on the di-electronic correlation $\mathrm{e}_{1}-\mathrm{e}_{2}$ through the potential $1 / r_{12}$. This means that the sole potential $-Z_{P} / s_{2}$ between $Z_{P}$ and $\mathrm{e}_{2}$ in the $T_{i f}$ can lead to capture of the electron $\mathrm{e}_{1}$, because of the underlying di-electronic correlation, which is inherently present in the e $e_{2}$ 's coordinate $\vec{s}_{2}$ through $\vec{r}_{12}$. In this paper we shall evaluate the role of the term $V\left(R, s_{2}\right)$ in the case of total as well as DCS. The second term in $U_{i}$ is a major perturbation $V_{o p} \equiv-\vec{\nabla}_{x_{1}} \ln \varphi_{i}\left(\vec{x}_{1}, \vec{x}_{2}\right) \cdot \vec{\nabla}_{s_{1}}$. Due to this term the corresponding $T$ matrix acquires a contribution from the two-centre function 


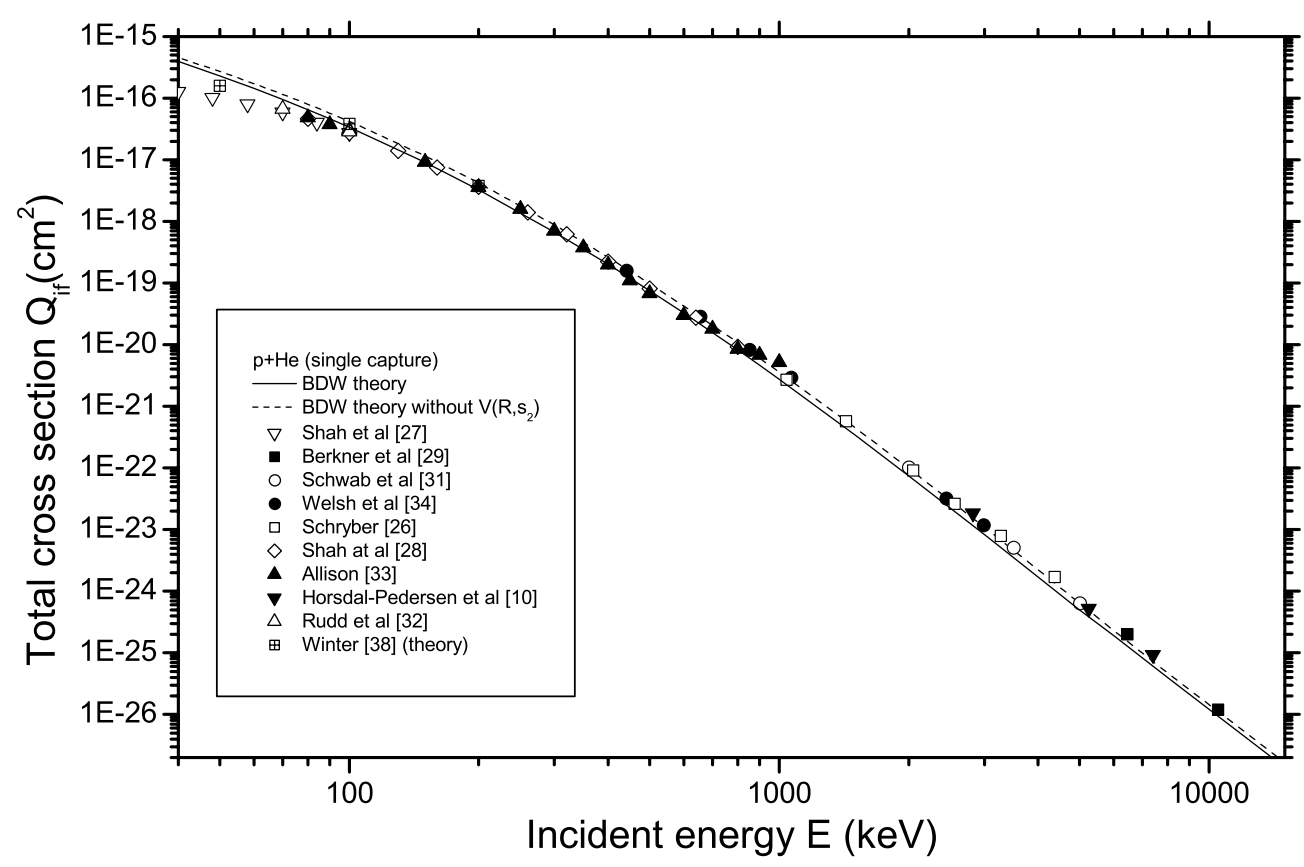

Figure 1. The total cross sections $Q_{i f}\left(\mathrm{~cm}^{2}\right)$ as a function of the laboratory incident energy $E$ $(\mathrm{keV})$ for the reaction: $\mathrm{p}+{ }^{4} \mathrm{He}\left(1 \mathrm{~s}^{2}\right) \longrightarrow \mathrm{H}(\Sigma)+{ }^{4} \mathrm{He}$. The symbol $\Sigma$ in $\mathrm{H}(\Sigma)$ means that the results obtained are multiplied by 1.202 in order to include the influence of all the excited states. The full line represents the cross sections $Q_{i f}$ of the present BDW-4B approximation. The broken curve refers to the BDW-4B results obtained without potential $V\left(R, s_{2}\right)=Z_{P}\left(1 / R-1 / s_{2}\right)$. The various experimental data are denoted in the figure by symbols.

$\sim \vec{\nabla}_{x_{1}} \varphi_{i}\left(\vec{x}_{1}, \vec{x}_{2}\right) \cdot \vec{\nabla}_{s_{1} 1} F_{1}\left(\mathrm{i} v_{P}, 1, \mathrm{i} v s_{1}+\mathrm{i} \vec{v} \cdot \vec{s}_{1}\right)$ which couples the bound state of $\mathrm{e}_{1}$ on $Z_{T}$ with its simultaneous continuum state in the field of $Z_{P}$.

The total cross sections are given by

$$
Q_{i f}\left(\pi a_{0}^{2}\right)=\frac{1}{2 \pi^{2} v^{2}} \int_{0}^{\infty} \mathrm{d} \eta \eta\left|T_{i f}\right|^{2} .
$$

In the present computations, following [36] we made a change of the variable $\eta$ in equation (3.2) such that the new variable $u$ effectively concentrates the integration points near the forward cone, i.e. $\eta=\sqrt{2(1+u) /(1-u)}$, where $u \in[-1,+1]$. The standard Cauchy regularization of the whole integrand is also accomplished before applying the universal Gauss-Legendre numerical quadratures.

Numerical computations of the total cross sections are presently carried out at impact energies ranging from $40 \mathrm{keV}$ to $15 \mathrm{MeV}$. The results obtained are shown in figure 1, where we compare the BDW-4B theory with a number of experimental data [10, 26-34]. As can be seen, the BDW-4B method is found to be consistently in excellent agreement with available experimental data for total cross sections from intermediate to high non-relativistic energies. Experimental data do not relate to process $\mathrm{p}+{ }^{4} \mathrm{He}\left(1 \mathrm{~s}^{2}\right) \longrightarrow \mathrm{H}(\Sigma)+{ }^{4} \mathrm{He}^{+}(1 \mathrm{~s})$, but rather to $\mathrm{p}+{ }^{4} \mathrm{He}\left(1 \mathrm{~s}^{2}\right) \longrightarrow \mathrm{H}(\Sigma)+{ }^{4} \mathrm{He}^{+}$, where the symbol ${ }^{4} \mathrm{He}^{+}$indicates that no information is available on the post-collisional state of the target remainder $\mathrm{He}^{+}$. This means that, if rigorous comparison with measurement is desired, the theory has to allow for all possible contributions arising from the transition of the non-captured electron $\mathrm{e}_{2}$ in $\mathrm{He}^{+}$. In practice, however, only excitation and ionization of the ion $\mathrm{He}^{+}$can play a non-negligible role at high 

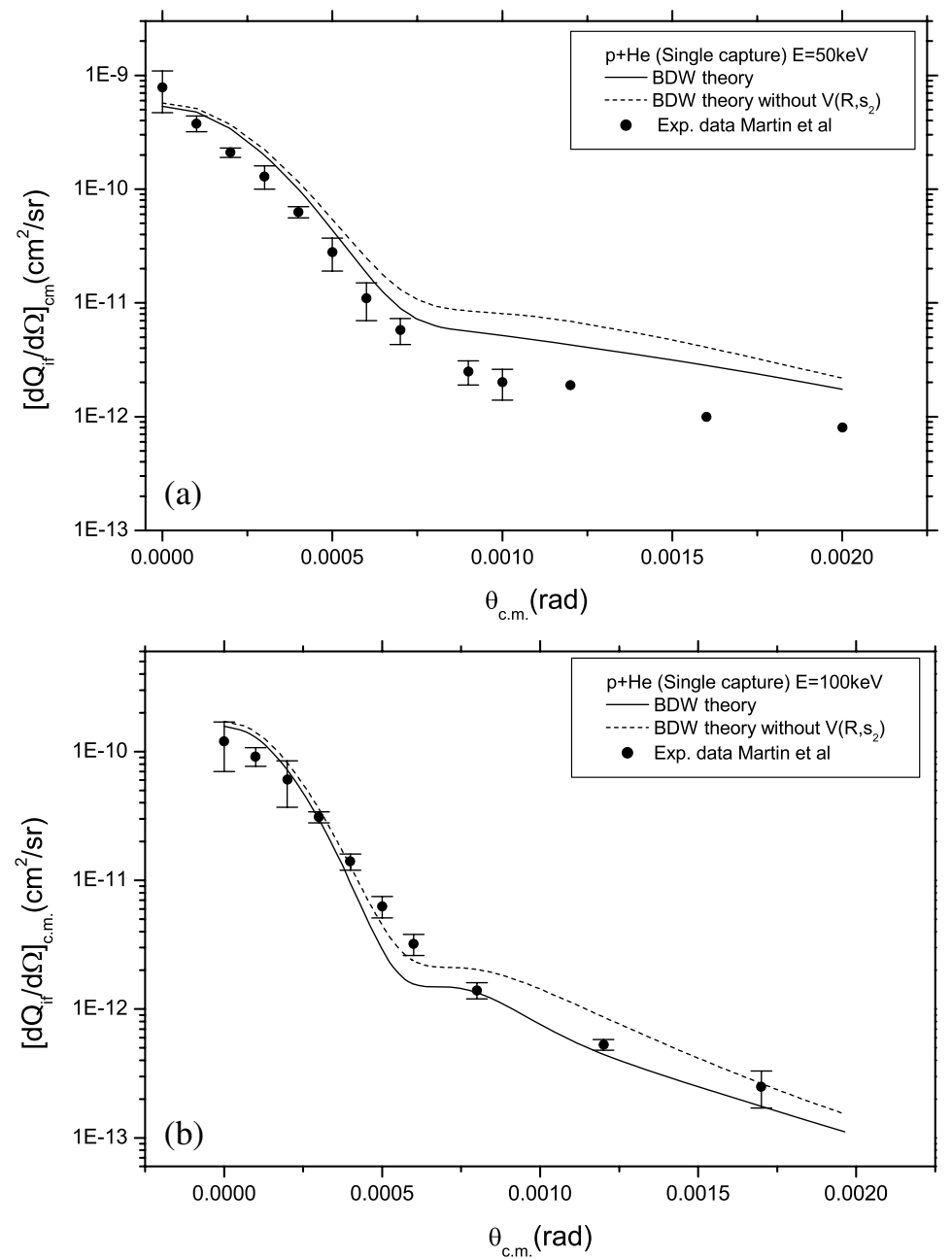

Figure 2. (a) The DCS d $Q_{i f} / \mathrm{d} \Omega\left(\mathrm{cm}^{2} \mathrm{sr}^{-1}\right)$ as a function of scattering angle $\theta$ (rad) for $50 \mathrm{keV}$ proton impact. Both cross sections and scattering angle are in the centre-of-mass system. Full curve: our BDW-4B results. Broken curve: our BDW-4B results obtained without the potential $V\left(R, s_{2}\right)=Z_{P}\left(1 / R-1 / s_{2}\right)$. Experimental data: [8]. (b) The same as in figure 2(a) except for $100 \mathrm{keV}$ proton impact.

impact energies. In the same figure, a comparison with the theoretical results of Winter [38] is made. The calculation of the cross sections in [38] was carried out for proton energies 50, 100 and $200 \mathrm{keV}$ by means of a coupled-state approach using bases of about 50 Sturmian functions. The full two-electron interaction was included. Agreement between our results and results of Winter [38] is quite good. The cross sections from the CDW-4B obtained by Belkić et al [13] (not shown in figure 1) are also in good agreement with our findings. Further, it is also important to investigate the post version of the BDW-4B approximation. An analogous derivation is carried out for the post form of the transition amplitude $\left(T_{i f}^{+}\right)$. Calculation of the post form is more difficult from the numerical point of view. Namely, the perturbation potential $U_{f}$ in the post transition amplitude is given by

$$
U_{f}=Z_{P}\left(\frac{1}{R}-\frac{1}{s_{2}}\right)-\left(\frac{1}{x_{1}}-\frac{1}{r_{12}}\right)-\varphi_{T}\left(\vec{x}_{2}\right) \vec{\nabla}_{s_{1}} \ln \varphi_{P}\left(\vec{s}_{1}\right) \cdot \vec{\nabla}_{x_{1}} .
$$




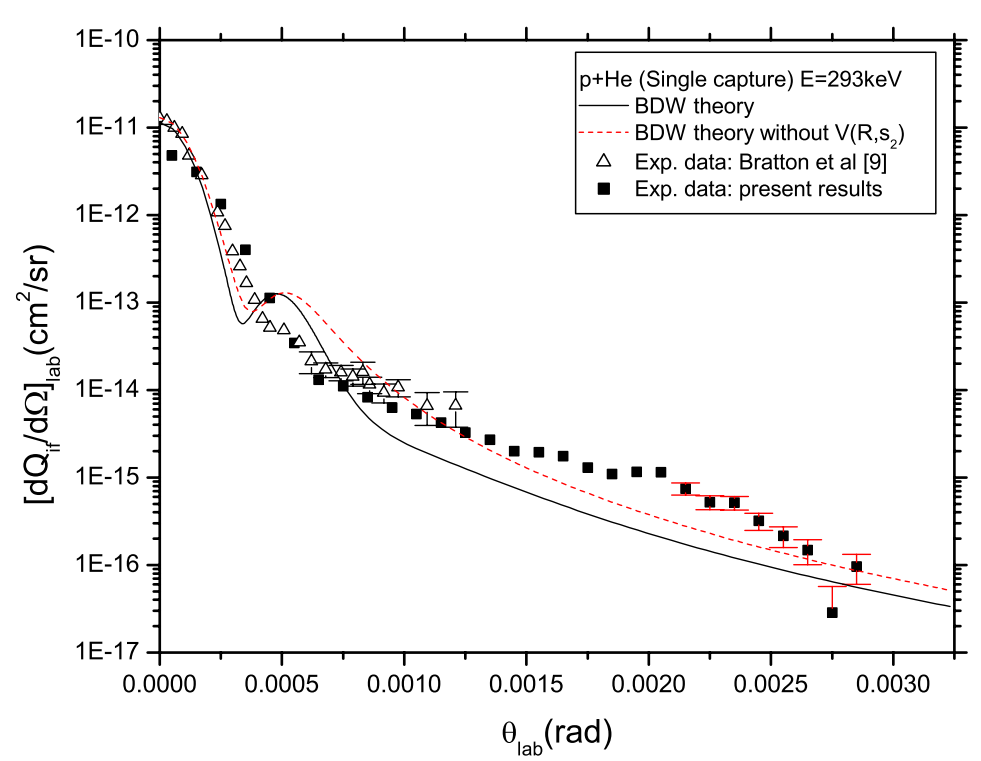

Figure 3. The DCS d $Q_{i f} / \mathrm{d} \Omega\left(\mathrm{cm}^{2} \mathrm{sr}^{-1}\right)$ as a function of scattering angle $\theta$ (rad) for $293 \mathrm{keV}$ proton impact. Both cross sections and scattering angle are in the laboratory system. Full curve: our BDW-4B results. Broken curve: our BDW-4B results obtained without potential $V\left(R, s_{2}\right)=Z_{P}\left(1 / R-1 / s_{2}\right)$. Experimental data: $\triangle,[9] ; \mathbf{\square}$, present results.

The term $1 / r_{12}$ in $U_{f}$ requires an additional three-dimensional integral in $T_{i f}^{+}$which must be evaluated numerically. Using a similar technique as in the prior form, the post total cross section can be analytically reduced to six-dimensional quadratures. At higher impact energies both prior $\left(Q_{i f} \equiv Q_{i f}^{-}\right)$and post $\left(Q_{i f}^{+}\right)$total cross sections are very close to each other. The significant difference appears only at lower energies. For example, the prior total cross sections at 40, 150 and $300 \mathrm{keV}$ are $3.9 \times 10^{-16}, 9.1 \times 10^{-18}$ and $6.8 \times 10^{-19} \mathrm{~cm}^{2}$, whereas the post values are $1.6 \times 10^{-16}, 7.2 \times 10^{-18}$ and $6.1 \times 10^{-19} \mathrm{~cm}^{2}$, respectively. The ratios $Q_{i f}^{-} / Q_{i f}^{+}$at these three energies are 2.4, 1.3 and 1.1. At lower energies post cross sections exhibit a slightly better agreement with experimental data. Notice that the dynamic electron correlation term $V\left(x_{1}, r_{12}\right)=1 / x_{1}-1 / r_{12}$ explicitly appears in the perturbation potential $U_{f}$ of the post transition amplitude. The residual potential $1 / x_{1}$ is the limiting value of $1 / r_{12}$ at infinitely large $x_{1}$ and finite $x_{2}$. It is clear now from equations (2.3) and (3.3) that use of the uncorrelated closed-shell $(1 \mathrm{~s})^{2}$ Slater-screened hydrogenic wavefunction, accompanied with the neglect of $V\left(R, s_{2}\right)$ and $V\left(x_{1}, r_{12}\right)$ in the case of the post form (i.e. neglect of $V\left(R, s_{2}\right)$ in the prior form), will effectively reduce the matrix elements of the BDW-4B method to those of its three-body counterpart. Hence, the proposed four-body formalism which neglects $V\left(R, s_{2}\right)$ and $V\left(x_{1}, r_{12}\right)$ in the post form $\left(V\left(R, s_{2}\right)\right.$ in the prior form) would be, in a sense, equivalent to the corresponding independent particle model.

A comparison of the theoretical results with experimentally determined DCS provides a more sensitive test of the theoretical models. The DCS in the BDW-4B model for $\mathrm{p}+\mathrm{He}$ collisions at 50 and $100 \mathrm{keV}$ are depicted in figures 2(a) and (b), together with the experimental data of Martin et al [8]. The calculation at $50 \mathrm{keV}$ yields results which slightly overestimate the experimental data at larger angles. At $100 \mathrm{keV}$ there is very good agreement with the measurements, as can be seen from figure 2(b). Bross et al [35] also measured DCS for single capture in $\mathrm{p}+\mathrm{He}$ collisions at $100 \mathrm{keV}$ and their results are very close to those of Martin et al [8]. 

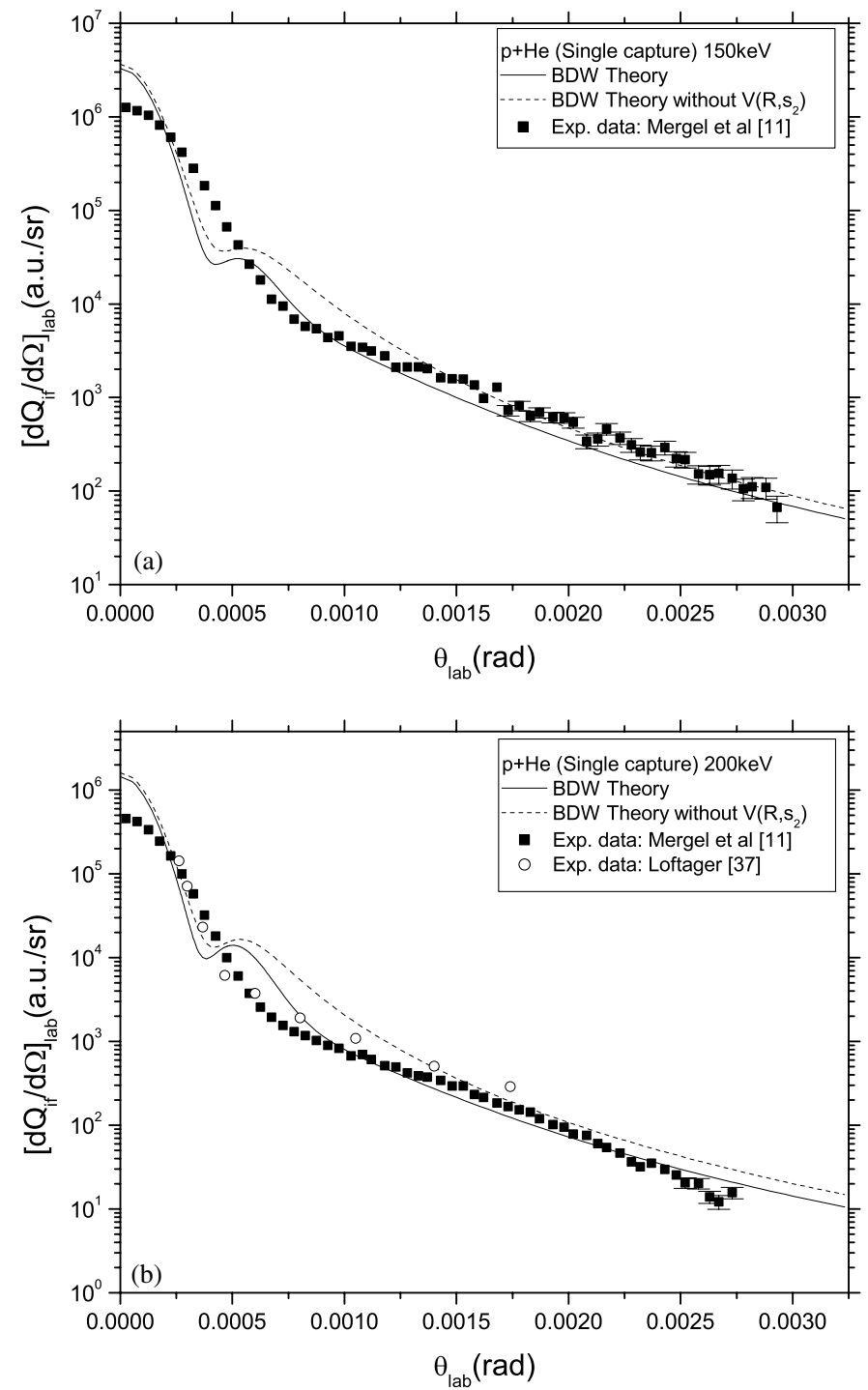

Figure 4. (a) The DCS d $Q_{i f} / \mathrm{d} \Omega\left(\mathrm{au} \mathrm{sr}^{-1}\right)$ as a function of scattering angle $\theta$ (rad) for $150 \mathrm{keV}$ proton impact. Both cross sections and scattering angle are in the laboratory system. Full curve: our BDW-4B results. Broken curve: our BDW-4B results obtained without the potential $V\left(R, s_{2}\right)=Z_{P}\left(1 / R-1 / s_{2}\right)$. Experimental data: $\mathbf{\square}$, [11]. (b) The DCS d $Q_{i f} / \mathrm{d} \Omega\left(\mathrm{au} \mathrm{sr}^{-1}\right)$ as a function of scattering angle $\theta(\mathrm{rad})$ for $200 \mathrm{keV}$ proton impact. Both cross sections and scattering angle are in the laboratory system. Full curve: our BDW-4B results. Broken curve: our BDW-4B results obtained without the potential $V\left(R, s_{2}\right)=Z_{P}\left(1 / R-1 / s_{2}\right)$. Experimental data: $\mathbf{\square}$, [11]; O, [37]. (c) The same as in (b) except for $300 \mathrm{keV}$ proton impact. (d) The same as in (b) except for $400 \mathrm{keV}$ proton impact.

The results from the BDW-4B method for the DCS at $293 \mathrm{keV}$ are shown in figure 3. The two experimental findings are depicted in the same figure. It is seen that good agreement is found. In figures 4(a)-(d) the DCS for proton energies 150, 200, 300 and $400 \mathrm{keV}$ are presented. A comparison is made with the experimental measurements of Mergel et al [11]. As can be seen from figures 4(a) and (b), there is good agreement at impact energies 150 and $200 \mathrm{keV}$. 

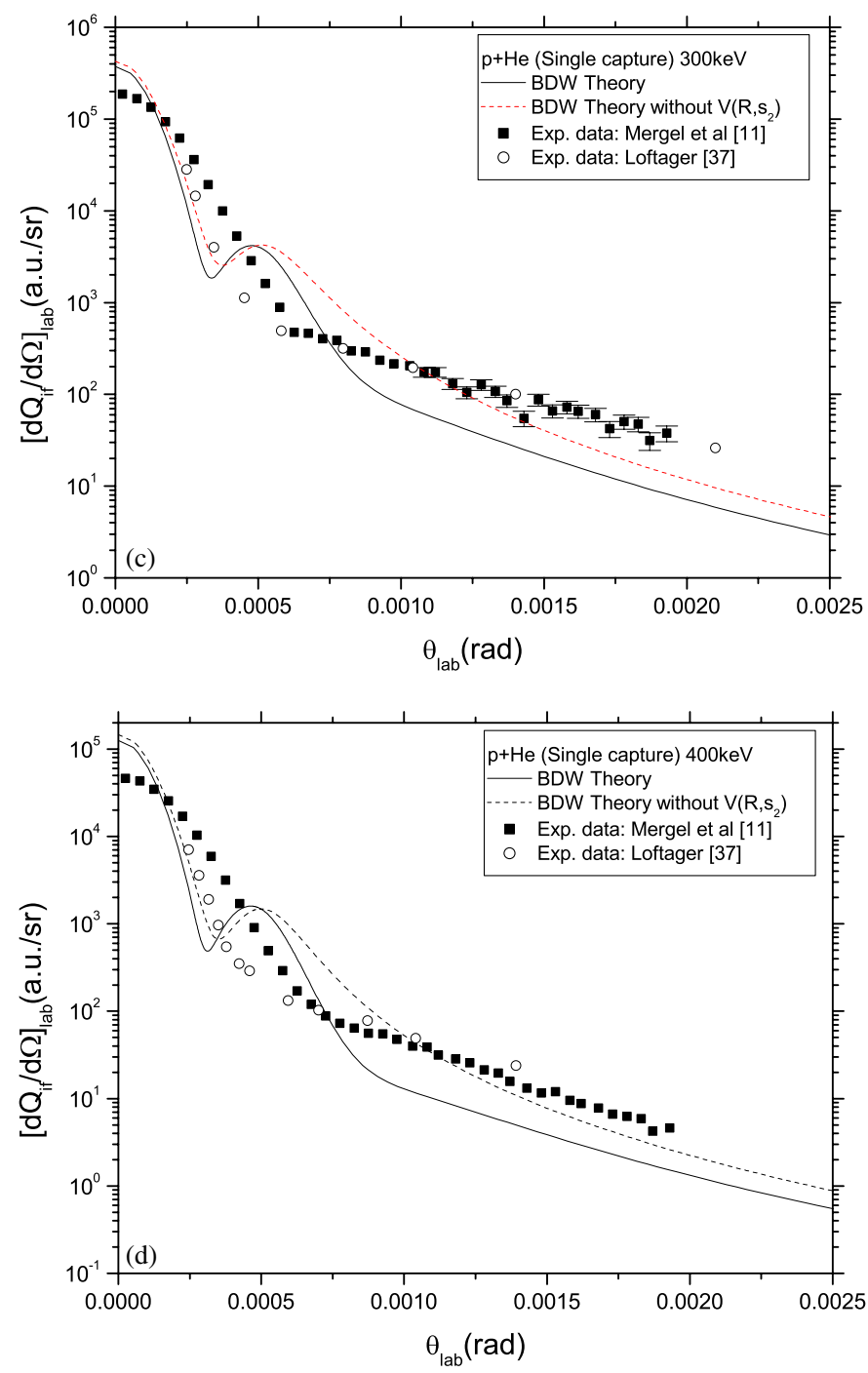

Figure 4. (Continued.)

Also at 300 and $400 \mathrm{keV}$ a satisfactory agreement is obtained. For the latter two energies, at larger scattering angles, the experimental data slightly overestimate the theoretical results, but otherwise both the theory and the experiment exhibit the same behaviour. We recall that the DCS in the BDW-4B method are obtained after performing two-dimensional numerical integrations, unlike the CDW-4B theory in which the five-dimensional quadratures are required for the same purpose.

Next we examine the role of the 'passive' electron by displaying the results with and without $V\left(R, s_{2}\right)$. In all the figures, the results from the BDW-4B method (broken curve) are obtained without the perturbation potential $V\left(R, s_{2}\right)$, i.e. only the contribution from the term $\left|T_{\nabla}\right|^{2}$ (see equation (2.5)) is taken into account. At all scattering angles the DCS without the term $V\left(R, s_{2}\right)$ is found to be larger than the DCS with the inclusion of the potential $V\left(R, s_{2}\right)$. 

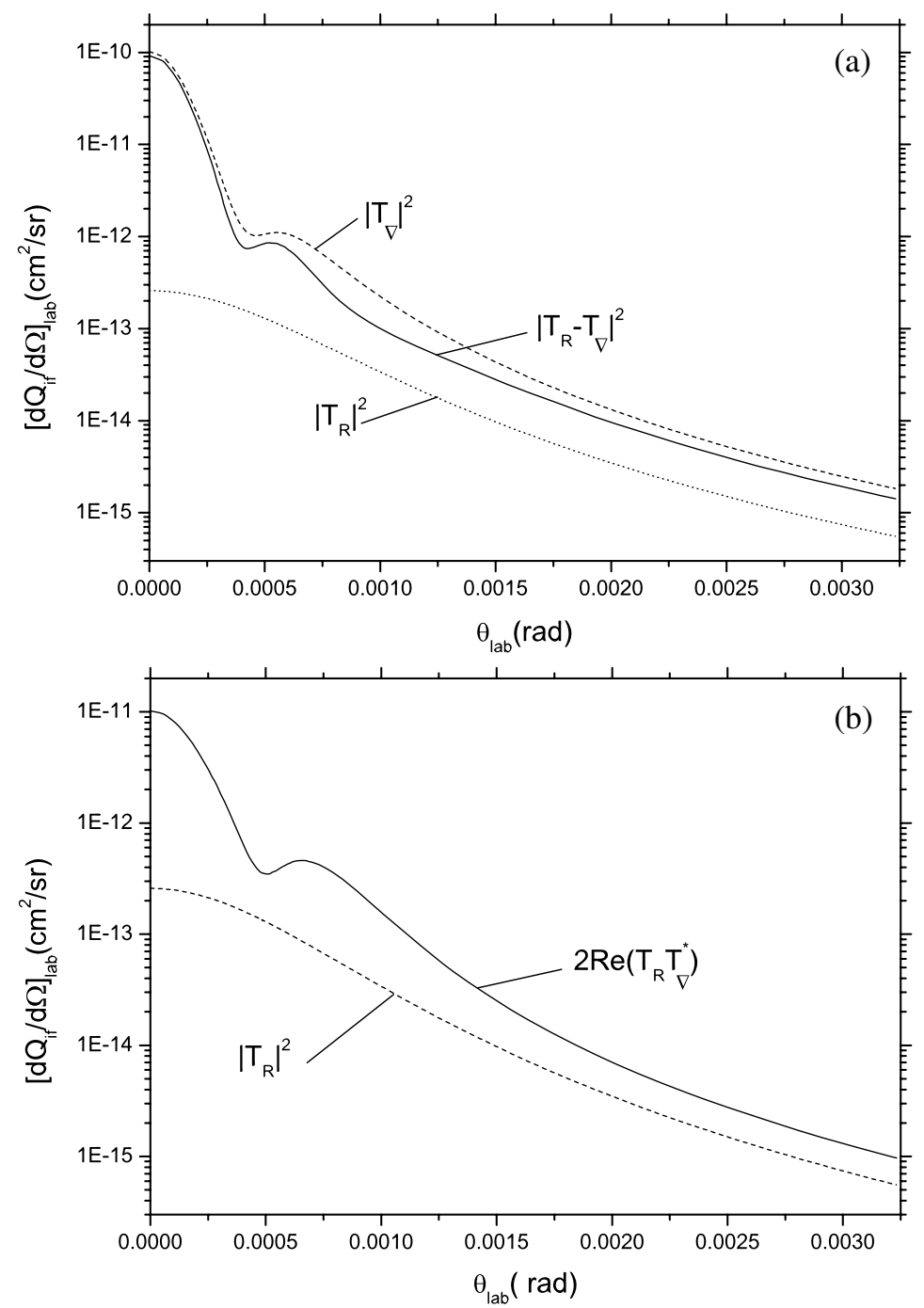

Figure 5. (a) Comparison of the DCS for charge transfer in $150 \mathrm{keV}$ proton-helium collisions calculated using equation (3.4). Full curve: our BDW-4B results obtained by means of $\mathrm{d} Q_{i f} / \mathrm{d} \Omega=$ $C\left|T_{R}-T_{\nabla}\right|^{2}$. Broken curve: our BDW-4B results obtained from $\mathrm{d} Q_{i f} / \mathrm{d} \Omega=C\left|T_{\nabla}\right|^{2}$. Dotted curve: our BDW-4B results obtained from $\mathrm{d} Q_{i f} / \mathrm{d} \Omega=C\left|T_{R}\right|^{2}$. The quantities $T_{R}$ and $T_{\nabla}$ are defined by equation (2.5). (b) Comparison of the DCS for charge transfer in $150 \mathrm{keV}$ protonhelium collisions calculated using equation (3.4). The full curve represents the DCS obtained using the BDW-4B method with the interference term $\mathrm{d} Q_{i f} / \mathrm{d} \Omega=2 C \operatorname{Re}\left(T_{R} T_{\nabla}^{*}\right)$. Dotted curve: our BDW-4B results obtained from $\mathrm{d} Q_{i f} / \mathrm{d} \Omega=C\left|T_{R}\right|^{2}$.

Obviously there is an interference between the two contributions $\left(T_{R}\right.$ and $\left.T_{\nabla}\right)$, since

$$
\frac{\mathrm{d} Q_{i f}}{\mathrm{~d} \Omega}=C\left|T_{R}-T_{\nabla}\right|^{2}=C\left\{\left|T_{R}\right|^{2}+\left|T_{\nabla}\right|^{2}-2 \operatorname{Re}\left(T_{R} T_{\nabla}^{*}\right)\right\}
$$

where $C=\left(\mu_{i} \mu_{f} / 2 \pi\right)^{2} \simeq\left(\mu_{P T} / 2 \pi\right)^{2}$. In order to evaluate a contribution from these terms, as illustrated in figure $5(\mathrm{a})$, we use the impact energy $150 \mathrm{keV}$ as a test and compare $\left|T_{R}-T_{\nabla}\right|^{2}$, $\left|T_{\nabla}\right|^{2}$ and $\left|T_{R}\right|^{2}$. Also in figure 5(b) a comparison between the interference terms $2 \operatorname{Re}\left(T_{R} T_{\nabla}^{*}\right)$ 
and $\left|T_{R}\right|^{2}$ is made. As can be seen from figures 5(a) and (b), the term $\left|T_{R}\right|^{2}$ is smaller than the other terms, especially at small scattering angles. A very similar dependence is observed for other impact energies considered in the present work. We can conclude that the contribution from the potential $V\left(R, s_{2}\right)$ in the DCS is negligible at small scattering angles. The difference between the results with and without the potential $V\left(R, s_{2}\right)$ at larger scattering angles is not so strongly apparent in the total cross sections, which are predominantly determined by collisions near the narrow forward direction. The conventional Thomas double scattering for reaction (3.1) takes place at $\theta_{l a b}=\left(1 / m_{P}\right) \sin 60^{\circ} \simeq 0.472 \mathrm{mrad}$, and the proposed BDW-4B theory shows a peak at this angle in the DCS above $100 \mathrm{keV}$, but the considered impact energies are too small to exhibit this effect clearly in the measurements. A very similar peak at the corresponding angle occurs in the theoretical results from [20,22] for double charge exchange in $\alpha+\mathrm{He}$ at $1.5 \mathrm{MeV}$ (i.e. $375 \mathrm{keV} \mathrm{amu}^{-1}$ ). It should be noted that none of the presented theoretical data for the DCS are folded with the experimental resolution functions.

\section{Conclusions}

We have investigated single charge exchange in $\mathrm{p}+$ He collisions by means of the BDW4B approximation. The BDW-4B method is a four-body theory which satisfies the correct boundary conditions in the entrance and exit channels for a general four-body rearrangement process (2.1). Unlike the CDW-4B method, which takes full account of the continuum intermediate states of the electron $\mathrm{e}_{1}$ in both channels, the BDW-4B method in the exit channel completely ignores these purely electronic ionization continua and accounts only for the Coulomb distortion phase $\exp \left\{\mathrm{i}\left[\left(Z_{P}-1\right)\left(Z_{T}-1\right) / v\right] \ln (v R+\vec{v} \cdot \vec{R})\right\}$ due to elastic scattering of the two scattering aggregates $\left(Z_{T} ; \mathrm{e}_{2}\right)$ and $\left(Z_{P} ; \mathrm{e}_{1}\right)$. In addition to the main interactions of the active electron $\mathrm{e}_{1}$, we have investigated the role of the potential $V\left(R, s_{2}\right)$ describing the two existing interactions of the non-captured electron $\mathrm{e}_{2}$. Overall, it is found that the interaction $V\left(R, s_{2}\right)$ yields a negligibly small contribution in the forward direction which predominantly determines the total cross section. Numerical computations of the total cross sections are carried out at impact energies from $40 \mathrm{keV}$ to $15 \mathrm{MeV}$. The theoretical results from the BDW-4B theory for total cross sections are in excellent agreement with the measurements. The computations of the DCS are performed at 50, 100, 150, 200, 293, 300 and $400 \mathrm{keV}$. For each of these energies, a comparison with experimental data is made and satisfactory agreement is obtained.

\section{Acknowledgments}

We are grateful to Professors Dž Belkić, H Schmidt-Böcking and R Dörner for helpful discussions and a critical review of the manuscript. I Mančev gratefully acknowledges financial support from the Ministry of Science and Technology of the Republic of Serbia through project no 1470 .

\section{References}

[1] Belkić Dž and Salin A 1978 J. Phys. B: At. Mol. Phys. 113905

[2] Rivarola R and Salin A 1984 J. Phys. B: At. Mol. Phys. 17659 Rivarola R, Salin A and Stockli M P 1984 J. Physique Lett. 45 L259

[3] Belkić Dž 1988 Europhys. Lett. 7323 Belkić Dž 1991 Phys. Rev. A 434751 
[4] Decker F and Eichler J 1989 J. Phys. B: At. Mol. Opt. Phys. 22 L95 Toshima N and Igarashi A 1992 Phys. Rev. A 456313

[5] Alston S 1990 Phys. Rev. A 42331

[6] McGuire J, Stockli M, Cocke C, Horsdal-Pedersen E and Sill N C 1984 Phys. Rev. A 3089

[7] Slim H, Heck E, Bransden B and Flower D 1991 J. Phys. B: At. Mol. Opt. Phys. 242353

[8] Martin P J, Arnett K, Blankenship D M, Kvale T J, Peacher J L, Redd E, Sutcliffe V C, Park J T, Lin C D and McGuire J 1981 Phys. Rev. A 232858

[9] Bratton T R, Cocke C L and Macdonald J R 1977 J. Phys. B: At. Mol. Phys. 10 L517

[10] Horsdal-Pedersen E, Cocke C and Stockli M 1983 Phys. Rev. Lett. 501910

[11] Mergel V, Dörner R, Khayyat Kh, Achler M, Weber T, Jagutzki O, Lüdde, Cocke C L and Schmidt-Böcking H 2001 Phys. Rev. Lett. 862257

[12] Mančev I 2003 J. Phys. B: At. Mol. Opt. Phys. 3693

[13] Belkić Dž, Gayet R, Hanssen J, Mančev I and Nuñez A 1997 Phys. Rev. A 563675

[14] Cheshire I M 1964 Proc. Phys. Soc. 8489

[15] Mančev I 1999 Phys. Rev. A 60351

[16] Mančev I 2001 Phys. Rev. A 64012708

[17] Belkić Dž 2001 J. Comput. Methods Sci. Eng. 11

[18] Mančev I 1995 Phys. Scr. 51768

[19] Mančev I 1996 Phys. Rev. A 54423

[20] Belkić Dž, Mančev I and Mudrinić M 1994 Phys. Rev. A 493646

[21] Belkić Dž 1994 Nucl. Instrum. Methods Phys. Res. B 8662

[22] Belkić Dž 1993 Phys. Rev. A 473824

[23] Belkić Dž, Gayet R and Salin A 1979 Phys. Rep. 56279

[24] Busnengo H F, Martinez A E, Rivarola R D and Dube L J 1995 J. Phys. B: At. Mol. Opt. Phys. 283283

[25] Galassi M E, Abufager P N, Martinez A E, Rivarola R D and Fainstein P D 2002 J. Phys. B: At. Mol. Opt. Phys. 351727

[26] Schryber U 1967 Helv. Phys. Acta 401023

[27] Shah M B, McCallion P and Gilbody H B 1989 J. Phys. B: At. Mol. Opt. Phys. 223037

[28] Shah M B and Gilbody H B 1985 J. Phys. B: At. Mol. Phys. 18899

[29] Berkner K H, Kaplan S N, Paulikas G A and Pyle R V 1965 Phys. Rev. 140729

[30] Williams J F 1967 Phys. Rev. 15797

[31] Schwab W, Baptista G B, Justiniano E, Schuch R, Vogt H and Weber E W 1987 J. Phys. B: At. Mol. Phys. 20 2825

[32] Rudd M E, DuBois R D, Toburen L H, Ratcliffe C A and Goffe T V 1983 Phys. Rev. A 283244

[33] Allison S 1958 Phys. Mod. Rev. 301137

[34] Welsh L M, Berkner K H, Kaplan S N and Pyle R V 1967 Phys. Rev. 15885

[35] Bross S W, Bonham S M, Gaus A D, Peacher J L, Vajnai T, Schulz M and Schmidt-Böcking H 1994 Phys. Rev. A 50337

[36] Belkić Dž 1988 Phys. Rev. A 3755

[37] Loftager P 2002 private communication

[38] Winter T 1991 Phys. Rev. A 444353

[39] Datta S K, Crothers D S F and McCarroll R 1990 J. Phys. B: At. Mol. Opt. Phys. 23479

[40] Gayet R 1972 J. Phys. B: At. Mol. Phys. 5483

[41] McCarroll R and Salin A 1967 Proc. Phys. Soc. 9063

[42] Belkić Dž 1989 Phys. Scr. T 28106

[43] Belkić Dž, Gayet R, Hanssen J and Salin A 1986 J. Phys. B: At. Mol. Phys. 192945

[44] Dewangan D P and Eichler J 1986 J. Phys. B: At. Mol. Phys. 192939

[45] Belkić Dž and Taylor H 1987 Phys. Rev. A 351991

[46] Belkić Dž and Gayet R 1977 J. Phys. B: At. Mol. Phys. 101923

[47] Deco G, Maidagan J M and Rivarola R D 1984 J. Phys. B: At. Mol. Phys. 17 L707

[48] Gravielle M S and Miraglia J E 1991 Phys. Rev. A 447299

[49] Deb N C and Crothers D S F 1989 J. Phys. B: At. Mol. Opt. Phys. 223725

[50] Busnengo H F, Martinez A E and Rivarola R D 1996 J. Phys. B: At. Mol. Opt. Phys. 294193 